\title{
CARoma Therapy: Pleasant Scents Promote Safer Driving, Better Mood, and Improved Well-Being in Angry Drivers
}

\author{
Dmitrijs Dmitrenko ${ }^{1}$, Emanuela Maggioni ${ }^{1}$, Giada Brianza ${ }^{1}$, \\ Brittany E. Holthausen ${ }^{2}$, Bruce N. Walker ${ }^{2}$, Marianna Obrist ${ }^{1}$ \\ ${ }^{1} \mathrm{SCHI}$ Lab, School of Engineering and Informatics, University of Sussex, Brighton, UK \\ ${ }^{2}$ Sonification Lab, Georgia Institute of Technology, Atlanta, GA, USA \\ 1 \{d.dmitrenko, e.maggioni, g.brianza, m.obrist $\} @$ sussex.ac.uk \\ $2\{$ brittany.holthausen, bruce.walker\}@psych.gatech.edu
}

\begin{abstract}
Driving is a task that is often affected by emotions. The effect of emotions on driving has been extensively studied. Anger is an emotion that dominates in such investigations. Despite the knowledge on strong links between scents and emotions, few studies have explored the effect of olfactory stimulation in a context of driving. Such an outcome provides HCI practitioners very little knowledge on how to design for emotions using olfactory stimulation in the car. We carried out three studies to select scents of different valence and arousal levels (i.e. rose, peppermint, and civet) and anger eliciting stimuli (i.e. affective pictures and on-road events). We used this knowledge to conduct the fourth user study investigating how the selected scents change the emotional state, well-being, and driving behaviour of drivers in an induced angry state. Our findings enable better decisions on what scents to choose when designing interactions for angry drivers.
\end{abstract}

\section{Author Keywords}

Perception; Smell; Odour Stimulation; Multimodal Interfaces; Notification Systems; In-Car User Interfaces; Emotions.

\section{CCS Concepts}

-Human-centered computing $\rightarrow$ Interaction techniques; Empirical studies in HCI;

\section{INTRODUCTION}

Drivers may experience a whole range of emotions. Nevertheless, research on emotions and their influence on driving is mainly focused around anger [35, 54, 23]. This is understandable, since anger promotes dangerous driving [21], becoming the key reason for road traffic accidents [26]. Anger leads to more errors [35, 22], stronger acceleration [36, 54], and higher mean speed [54] than e.g. in the neutral state. For this reason, interventions for reducing anger should be a research priority.

Permission to make digital or hard copies of all or part of this work for personal or classroom use is granted without fee provided that copies are not made or distributed for profit or commercial advantage and that copies bear this notice and the full citation on the first page. Copyrights for components of this work owned by others than ACM must be honored. Abstracting with credit is permitted. To copy otherwise, or republish, to post on servers or to redistribute to lists, requires prior specific permission and/or a fee. Request permissions from permissions@acm.org.

CHI '20, April 25-30, 2020, Honolulu, HI, USA.

2020 Association of Computing Machinery.

ACM ISBN 978-1-4503-6708-0/20/04 \$ \$15.00.

http://dx.doi.org/10.1145/3313831.3376176
No other human sense has such a strong link to emotions as the sense of smell [2]. Scents can improve our mood [29] and help us relax [32]. Car manufacturers (e.g. Mercedes-Benz [14], BMW [7], and Bentley [6]) have already started including olfactory interfaces in their high-end vehicles to increase the well-being of the driver and passengers. Nevertheless, the choice of scents, the elicitation of specific emotions, and the impact of olfactory stimulation on the driving behaviour in these products are unknown. Also, the academic research offers only very few insights on the influence of scents on emotions in the driving process $[4,53]$. This issue may lead to designers choosing the wrong scent for a specific type of driving behaviour. To start tackling this problem, we define the following research question: Scents of what arousal and valence levels can reduce the negative emotion of angry drivers? The key contributions of our paper are summarised below:

- Mapping different scents on the arousal/valence circumplex, covering different quadrants of this two-dimensional space.

- Identifications of anger-eliciting IAPS (International Affective Picture System) [39] pictures.

- Identification of anger-eliciting on-road events.

- First empirical evidence for the positive effect of high/low arousal scents on reducing drivers' negative emotions.

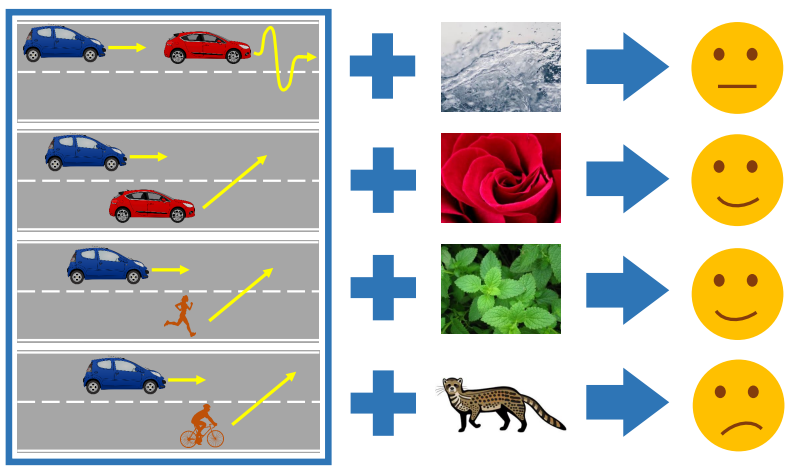

Figure 1: After having experienced anger-inducing on-road events (e.g. a car cutting off), drivers describe the valence of their emotional state as neutral in the water (clean air) condition, as positive in the rose and peppermint conditions, and as negative in the civet condition. 


\section{RELATED WORK}

We structured the related work around three research areas:

\section{Emotions While Driving}

Following the method proposed by Wilson et al. [62], we looked into the Emotions Circumplex established by Russell [55]. This model helped us gain an understanding of a wider spectrum of emotional labels and their mapping on the four quadrants of the arousal and valence dimensions, to tackle the problem of analysing driver's emotions more efficiently. Anger is an emotions that lies in the "high arousal, negative valence" quadrant. Roidl et al. [54] have found that anger leads to stronger acceleration and higher speed. Jeon et al. showed that anger also leads to more errors [36] and a degraded driving performance [35]. Chan and Singhal [12] demonstrated that distraction charged with negative emotions leads to reduced lateral control and slowed driving speed. Hayley et al. [26] showed that poor emotional control may impede the ability to drive safely. Finally, Eherenfreund-Hager et al. [22] confirmed that the arousing negative affect leads to increased risky driving. This area of research shows how negative emotional states (e.g. anger) are tied to negative driving behaviour. Therefore, minimising these negative emotions is of utmost importance. Olfactory displays are promising for this application due to the strong connection between emotions and scents [2].

\section{Stimulating Different Senses}

Prior research on emotions has mainly demonstrated that it is possible to improve the driving performance using visual and auditory stimulation. For example, displaying positive words to participants during the driving resulted in a decreased lane deviation [12]. Drivers who listened to either happy or sad music made significantly fewer errors than those who did not listen to music [23]. It has also been shown that participants drove significantly slower after the music had faded from the front to rear speakers [11].

Positive impact on the driver's emotions can also be achieved by using tactile stimulation. For example, vibrations in the driver's seat have been demonstrated to be less annoying and more appropriate in collision warnings studies [40].

Olfactory stimulation is still little explored [19], despite its potential benefits (e.g. a link to emotions and memories [28]).

\section{The Use of Scents While Driving}

Prior studies have revealed that scents can improve drivers' braking performance [43] and have a positive impact on the alertness and emotions of the driver [4, 53]. Moreover, olfactory stimulation has been proven to have a positive effect on keeping drowsy drivers awake $[66,24,30,51,65]$. Scents have also been demonstrated to be useful for conveying drivingrelated information $[19,18,16]$.

Initial findings on the effect of scents on drivers' emotions suggest that the scent of lemon results in a significant increment of participants' positive affect [4], and scents of peppermint and cinnamon reduce frustration [53]. Thus far, there are no studies that aim to determine if olfactory displays can be used to reduce negative emotional states while driving. Such a study is, however, essential to help HCI practitioners make informed decisions regarding the choice of scents when designing for driver's emotions.

In summary, while the olfactory interface and experience design, especially in an automotive context, comes with challenges (e.g. confined space, lingering, interpersonal differences [15]), we can build on advances in the understanding of olfaction as an interaction modality $[16,42]$ and advances in scent-delivery technology [15]. Latest techniques of scent delivery and extraction $[18,15]$ have shown that it is possible to enable quick detection of scents by the driver (10s), short lingering time (9s), and rapid switching between scents (just $19 \mathrm{~s}$ is enough to neutralise the previous scent).

In the next sections, we will present the process of choosing scents and anger eliciting visual stimuli. We will then explain how these olfactory and visual stimuli were used to study the participants' emotions, well-being, and driving behaviour.

\section{OVERVIEW OF STUDIES}

This paper presents the total of four studies investigating the effects of scents on drivers in an induced angry state:

- Study 1: Mapping different scents on the arousal/valence circumplex (i.e. identifying suitable scents).

- Study 2: Identifying the anger-eliciting pictures among the negative valence and high arousal IAPS [39] pictures.

- Study 3: Identifying anger-eliciting on-road events. Studies 2 and 3 have been conducted to identify the suitable angereliciting stimuli for the main study (i.e. Study 4).

- Study 4: Investigating the effects of different arousal and valence scents on emotions, well-being, and the driving behaviour of participants in an induced angry state.

All four studies were approved by the the Ethics Committee of the University of Sussex. Details of these studies are presented below. All participants were carefully screened to make sure they have no respiratory problems, no scent allergies, no adverse reactions to scents, and that they are not pregnant.

\section{STUDY 1: MAPPING SCENTS}

We initially conducted an exploratory scent rating study during a public science fair (like in [13]) in which we collected data on valence and arousal of 11 different scents (i.e. black pepper, cedarwood, eucalyptus, juniper, lemon, patchouli, peppermint, pine, rose, vanilla, and ylang-ylang). However, as the results of this study were not conclusive (i.e. no strict borders between valence and arousal quadrants) and not from a lab-based exploration, we decided to validate them in Study 1.

The aim of Study 1 was to validate the mapping of the scents of rose, peppermint, and patchouli on the valence and arousal quadrants. To add a "negative valence, high arousal" scent, we included a civet scent (fragrance oil from Plush Folly Ltd) [5].

The idea of this study was not to select specific scents but to pick one scents from each cluster of scents (i.e. arousal and valence quadrant) for further investigation. 


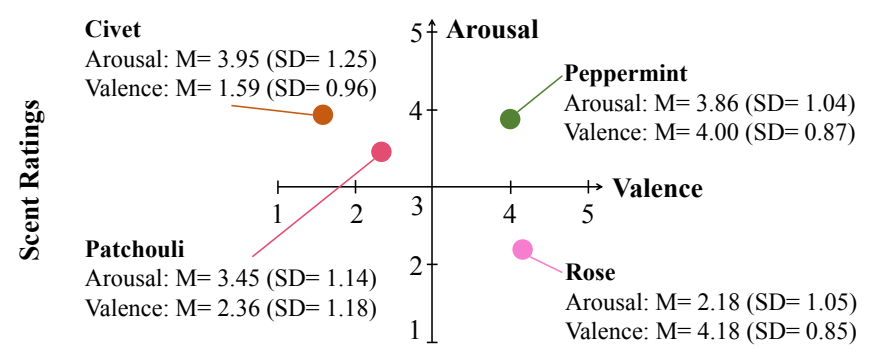

Figure 2: Mean Arousal (1= "Low", 5= "High") and Valence (1= "Negative", $5=$ "Positive") ratings of fours scents.

\section{Design}

We conducted a within-participants study asking the participants to rate four scents (peppermint, rose, patchouli, civet).

\section{Setup}

Participants sniffed the scents from identical bottles located on a table. Each bottle contained $10 \mathrm{ml}$ of essential oil. They sniffed each bottle for $2 \mathrm{~s}$, with intervals of 20 s (as in $[37,60]$ ).

\section{Procedure}

After sniffing each scent, participants were asked to rate the valence and arousal of each scent using SelfAssessment Manikins (SAM) [8] on a 5-Point Likert scale (1= "low/negative”, 5= "high/positive”, see Figure 2).

\section{Results}

22 participants, 20-38 years old $(M=28.68, S D=5.38,6$ females) volunteered for this study.

A normality test showed that the scent ratings were normally distributed. We did a repeated measures ANOVA test to analyse the data and found a main effect of scents on the arousal $(F(3,19)=11.26, p<.001 ;$ Wilks' $\lambda=.360)$ and valence $(F(3$, $19)=39.86, p<.001 ;$ Wilks' $\left.^{\prime} \lambda=.137\right)$ ratings. The results of this study (see Figure 2) confirmed that the scent of peppermint has positive valence and high arousal, the scent of rose has positive valence and low arousal, and the scent of civet has negative valence and high arousal. Only patchouli was not rated as expected, landing in the same quadrant as civet.

\section{STUDY 2: IDENTIFYING ANGER-ELICITING PICTURES}

Related work suggests such approaches of inducing emotions in participants as re-visiting an emotional situation from the past $[36,35]$, showing videos $[23,56]$, presenting emotionally charged words on traffic signs [22], delivering emotionally charged voice signals during the driving [12], and asking participants to imagine they were late on their way to work [54].

We used IAPS to elicit anger due to three reasons:

1. driving is a highly visual task, and visual stimuli have already been successfully applied for eliciting anger in simulated driving studies in the past $[23,56,22]$,

2. the effect of IAPS is comparable with auditory stimuli [41],

3. with IAPS we can be sure that we will elicit an emotion of the required arousal and valence levels, as it has been validated through a multitude of studies in HCI (e.g. [61]).
IAPS provides information on the arousal, valence, and dominance ratings of each picture. However, it is not clear which "high arousal, low valence" pictures specifically elicit anger. With this study, we were able to find this out.

\section{Design}

We conducted a within-participants study in which each participant had to observe and rate ten different IAPS pictures.

\section{Setup}

With a few exceptions for the pictures showing traffic accidents, most of the IAPS pictures are not related to driving. We chose ten negative valence and high arousal pictures (see Table 1) to be rated on a computer screen.

\section{Procedure}

Participants saw each picture for $5 \mathrm{~s}$ and rated them by answering the following question: "How angry does this picture make you feel?" ( $1=$ "Not angry at all", 7= "Very angry").

\section{Results}

28 participants, $23-53$ years old $(M=30.25, S D=5.92,16$ females) volunteered for this study. A Shapiro-Wilk test showed a significant departure from normality for all the variables. We conducted a Friedman test and found a statistically significant difference in the effect of pictures on anger ratings $\left(\chi^{2}(9)=\right.$ 99.7, $p<.001)$. Three pictures with the highest mean anger ratings (i.e. \#6212,\#6313, and \#9410) were chosen to induce anger in participants before the driving task (see Table 1).

\begin{tabular}{|l|l|c|}
\hline Picture & Description & $\begin{array}{c}\text { Anger rating: } \\
\text { M (SD) }\end{array}$ \\
\hline$\# \mathbf{6 2 1 2}$ & Soldier pointing a gun at a child & $\mathbf{6 . 0 7}(\mathbf{1 . 6 7})$ \\
\hline$\# \mathbf{6 3 1 3}$ & Man attacking a woman & $\mathbf{5 . 9 0}(\mathbf{1 . 6 5})$ \\
\hline$\# 6563$ & Gun pointed at a teenager's head & $5.55(1.74)$ \\
\hline$\# 9040$ & Person suffering from starvation & $5.59(1.97)$ \\
\hline$\# 9250$ & Medical staff with an injured person & $4.52(2.16)$ \\
\hline$\# 9410$ & A man carrying an injured child & $\mathbf{6 . 0 0}(\mathbf{1 . 7 9})$ \\
\hline$\# 9413$ & Two men about to be hanged & $5.55(1.76)$ \\
\hline$\# 9635 \_1$ & Person being set on fire & $5.62(1.90)$ \\
\hline$\# 9908$ & Traffic accident & $3.90(2.04)$ \\
\hline$\# 9921$ & Fire-fighters saving a person from fire & $4.28(2.09)$ \\
\hline
\end{tabular}

Table 1: IAPS pictures studied to establish anger-eliciting stimuli, with the corresponding ratings. Three pictures that got selected for the main study are highlighted in bold.

\section{STUDY 3: IDENTIFYING ANGER-ELICITING EVENTS}

Besides inducing anger in participants before the driving phase, it is important to keep them angry throughout the driving. We conducted a study to investigate which on-road events make drivers angry. The related work showed that displaying emotional stimuli on signs [22] and making drivers wait [54] helps keep them angry. We wanted to explore further scenarios.

\section{Design}

We ran a between-participants study with two conditions. In the first condition, participants experienced 12 different angereliciting on-road events. In the second condition, before experiencing the 12 anger-eliciting events, participants also viewed three IAPS pictures (see Table 1) before the driving phase. 


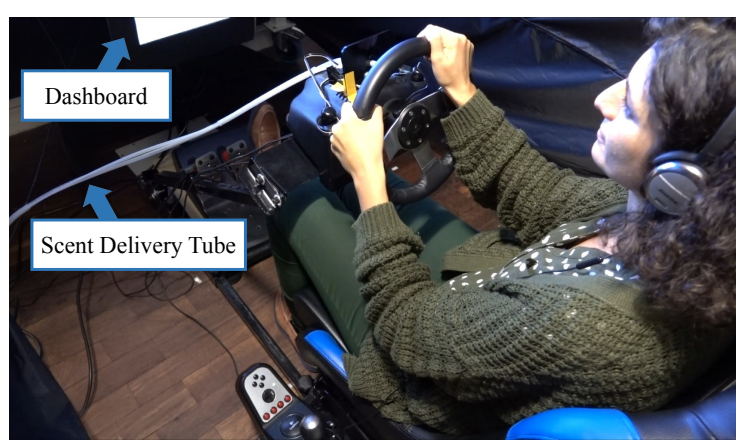

Figure 3: Participant sitting in the driving simulator.

\section{Setup}

We developed the course using the IPG CarMaker software. The participants were sitting in a driving simulator seat $(F K$ Automotive) equipped with the Logitech G27 steering wheel in front of the main screen $(55$ ", $60 \mathrm{~Hz}$ refresh rate), on which the view outside the car from driver's position was rendered. The dashboard was presented on an additional screen $(17$ ', $60 \mathrm{~Hz}$ refresh rate), in front of the main screen (see Figure 3).

\section{Procedure}

In the condition with IAPS pictures shown before the driving, pictures were shown on the simulator's main screen, in its full height. Pictures appeared in a randomised order, for $5 \mathrm{~s}$ each.

In both conditions, during the driving phase, there were no anger-eliciting events in the first 90 s, so that participants could become familiar with the simulator [52]. After this stage, one of the 12 events (see Table 2 ) took place every 30 s.

\begin{tabular}{|l|c|c|}
\hline \multirow{2}{*}{ Event } & \multicolumn{2}{|c|}{$\begin{array}{c}\text { Anger rating } \\
\text { in two conditions: }\end{array}$} \\
\cline { 2 - 3 } & $\mathbf{1 :}$ M (SD) & $\mathbf{2 : ~ M ( S D )}$ \\
\hline IAPS picture \#6212 on a billboard & $4.86(1.95)$ & $3.86(1.68)$ \\
\hline IAPS picture \#6313 on a billboard & $5.14(2.27)$ & $4.29(1.80)$ \\
\hline IAPS picture \#9410 on a billboard & $4.14(2.12)$ & $4.86(1.35)$ \\
\hline 30s waiting time at a traffic light & $3.14(1.35)$ & $2.57(1.13)$ \\
\hline Car cutting off & $5.43(2.07)$ & $\mathbf{5 . 2 9}(\mathbf{2 . 0 6})$ \\
\hline Slow zigzagging lead vehicle & $4.57(2.30)$ & $\mathbf{5 . 7 1}(\mathbf{2 . 2 1})$ \\
\hline Cyclist cutting off & $5.14(2.19)$ & $\mathbf{5 . 0 0}(\mathbf{1 . 5 3})$ \\
\hline Pedestrian suddenly crossing the road & $4.00(2.31)$ & $\mathbf{5 . 0 0}(\mathbf{1 . 5 3})$ \\
\hline Child playing with a ball on the street & $3.86(1.68)$ & $2.43(1.13)$ \\
\hline Sheep suddenly appearing on the road & $3.86(1.68)$ & $2.29(1.89)$ \\
\hline Unmarked roadworks site & $3.57(1.72)$ & $3.57(2.23)$ \\
\hline 30mph limit on a straight rural road & $4.29(1.38)$ & $3.71(2.36)$ \\
\hline Overall experience & $4.43(1.81)$ & $4.57(1.81)$ \\
\hline
\end{tabular}

Table 2: Anger-eliciting on-road events studied to select stimuli for the driving phase of the main study in two conditions: (1) without and (2) with anger-eliciting IAPS pictures shown to the participants prior to the driving phase. Events chosen for the main study are highlighted in bold.

After the driving phase, the participants were asked to rate how angry each event, and the drive as a whole, made them feel, on a 7-Point Likert scale ( $1=$ "Not angry at all", 7= "Very angry”). Such a rating procedure has been chosen in order not to interrupt the driving task [58]. This study took 10 minutes.

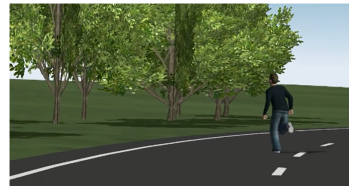

(a) Erratic pedestrian

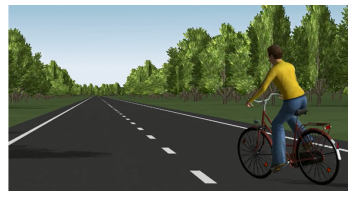

(c) Cyclist cutting off

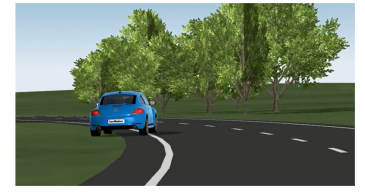

(b) Zigzagging car

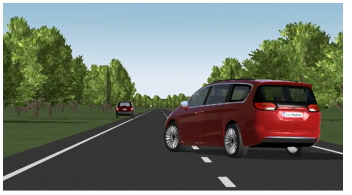

(d) Car cutting off
Figure 4: Four anger-inducing on-road events: (a) a pedestrian suddenly crossing the road, (b) a slow zigzagging vehicle that needs to be overtaken, (c) a cyclist cutting off, (d) a car cutting off.

\section{Results}

14 participants (seven per condition, three females in each) in the age of 23-43 years old $(M=30.14$ years, $S D=6.06)$ volunteered for this study. Participants' driving experience varied between 2 and 23 years $(M=8.93, S D=6.18)$.

We ran a normality test and found that data was normally distributed. After confirming the normality, an independent t-test was conducted to determine if there was a difference in average anger ratings between the two conditions (IAPS pictures followed by on-road events and just on-road events). The results showed no significant differences in the anger ratings of the different events between the two conditions and also within each condition.

As the anger rating of the overall driving experience was slightly higher for the condition where the IAPS pictures were displayed before the driving, we decided to use this approach in the main study and exclude pictures from the billboards.

For further exploration, we decided to use only the four most highly rated events (see Table 2, highlighted in bold): a car cutting off, a slow zigzagging lead vehicle, a cyclist cutting off, and a pedestrian suddenly crossing the road.

\section{STUDY 4: INVESTIGATING THE EFFECTS OF SCENTS}

In Studies 1-3, we chose a set of scents representing different quadrants of the arousal/valence dimensions, a set of angerinducing IAPS pictures, and a set of anger-inducing events.

\section{Design}

This study followed a 1 (emotional state: anger $) \times 4$ (scents: rose, peppermint, civet, water/clean air as a control stimulus) mixed model experimental design, with three main steps:

1. Familiarisation with the driving simulator,

2. Inducing anger in participants by displaying three emotionally charged IAPS [39] pictures (see Table 1).

3. Driving through a course composed of anger-inducing events (see Figure 4) under an effect of one of the four scents (rose, peppermint, civet, or water/clean air) 


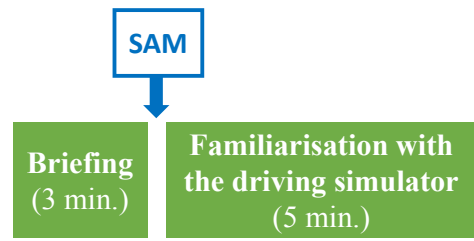

(1)
(2)

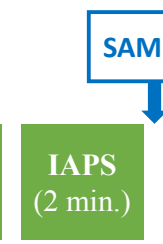

(3)

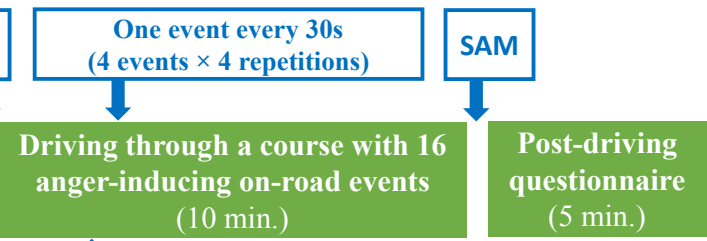

(4)
Interview +

Debriefing

$(5$ min.)

Scent of the condition delivered for $5 \mathrm{~s}$ (10s before each event)

Figure 5: The timeline of the Study 5 procedure.

We used the mean lane deviation (vehicle's deviation from the centre of the lane in metres) as a measure of the participants' driving performance. The mean steering angle (in radians), the average speed (in $\mathrm{mph}$ ), and the number of collisions were used to assess the driving behaviour. We also analysed the catastrophic road excursions (as per Mok et al. [46]) - events of the ego car leaving the road.

\section{Setup}

Driving Simulator

Here, we used the same driving simulator setup as in Study 3. The course was built using the IPG CarMaker software, based on the four anger-inducing events extracted from Study 3. The software used to log the driving data (using the CarMaker $A P I)$ and to control the scent-delivery device was written in $\mathrm{C}$.

\section{Scent-Delivery Device}

For this study, we have assembled and used a scent-delivery device (as per [15]). The study was conducted in an olfactory interaction room (as per [15]).

Our scent-delivery device contained four scent chambers. The first two were filled with $6 \mathrm{~g}$ of $100 \%$ pure essential oil of rose and peppermint (from Holland \& Barrett Int. Ltd.), chamber three contained $6 \mathrm{~g}$ of the civet scent (from Plush Folly Ltd), and chamber four was filled with $6 \mathrm{~g}$ of water (odourless water used as a neutral stimulus). The scent was delivered with the air pressure of 0.5 bar.

The output of the scent-delivery device was located behind the steering wheel and pointed towards the participants' face (as in [18]). Participants wore headphones playing the engine sound to cancel any potential external sounds.

\section{Procedure}

In this section, we will cover the procedure (see Figure 5) of this study, which consisted of the following seven steps:

\section{Briefing}

Upon arrival, participants were given the information sheet, driving instructions, and a consent form to sign. The driving instructions contained clear guidance regarding the driving rules, such as the speed limits. Participants were then asked to rate their current emotional state using the SAM [8] questionnaire and take a seat in the driving simulator.

\section{Familiarisation With the Driving Simulator}

Participants then became familiar with the driving simulator by driving on a rural highway for approximately five minutes.

\section{Inducing Anger Using IAPS Pictures}

Next, participants were shown three IAPS pictures. Participants were provided information about the graphic content in the Information sheet given at the beginning of the study. The three IAPS pictures (see Table 1) were displayed taking the full height of the screen. Each picture appeared for $5 \mathrm{~s}$, and the order of their presentation was randomised. After this, participants were again asked to rate their emotional state by filling in the SAM questionnaire.

\section{Driving Through an Anger-Inducing Course}

After the anger induction stage, participants were asked to drive straight, through a rural setting, respecting the speed limits (40-60 mph, shown on signs), until the simulation ended. They were instructed to expect traffic on the road, but no information on critical events was given. The course consisted of four anger-eliciting events (see Figure 4). One of these events appeared every 30s after the start of the driving phase. Each event was repeated four times (16 events in total), and the order of their occurrences was randomised. Ten seconds before each event, a scent of the condition was released, which is the time necessary for the scent to get perceived by the user in such a setup, as suggested by [15]. Each scent-delivery event lasted five seconds, in order to ensure that participants inhaled each scent [50]. Each participant received only one scent (a between-participants condition): rose, peppermint, civet, or clean air (control stimulus). As the events appeared every 30 s, the scent-delivery frequency was 30 s too, enough to neutralise the previously delivered scent $[15,49]$.

\section{Post-Driving Questionnaire}

After the driving phase, participants were asked to rate their emotional state one more time by completing the SAM questionnaire. Participants were also asked to answer questions on the liking, comfort, and intensity of the scent (as per [18]), as well as about their demographic data.

\section{Post-Driving Interview}

The experiment finished with a short semi-structured interview conducted to gain additional insights about how the participants felt. The interview was audio-recorded and structured around the following four questions:

1. How did the pictures make you feel?

2. How did the on-road events make you feel?

3. How were your emotions influenced by the scent?

4. How was your driving behaviour influenced by the scent? 


\section{Debriefing}

After the end of the interview, participants received a Debriefing Sheet and a $£ 5$ Amazon Voucher for their participation. Overall, the study lasted about 30 minutes.

\section{Results}

In this section, we summarise our results on the participants' emotions, driving performance/behaviour, negotiation of critical events, and their experience with the scents.

\section{Participants}

A total of 40 participants were recruited for the study (10 per condition, four females in each). The age ranged from 19 to 59 years $(M=30.73, S D=9.11)$. Their reported driving experience ranged from 1 to 41 years $(M=9.28, S D=9.07)$.

\section{Emotions Before Driving}

A Shapiro-Wilk test showed a significant departure from normality for all the variables. We run a Wilcoxon-Signed-Ranks test to compare the means between two variables. We compared the valence of the emotions before the experiment with its value after the IAPS pictures were shown. The same comparison was done for the corresponding arousal ratings. Concerning the valence ratings, the test indicated that the valence before the experiment (mean rank $=17.52$ ) was significantly higher than after viewing the IAPS pictures (mean rank= 11.00, $Z=-4.058, p<.001)$. Concerning the arousal ratings, the test indicated that the arousal before the experiment (mean rank $=10.33$ ) was statistically lower than after viewing the IAPS pictures (mean rank $=13.22, Z=-2.611, p<.01$ ).

This means, at the start of the study, all participants were calm (as per [55]). After viewing the IAPS pictures, participants' self-reported emotional state shifted towards the negative valence and high arousal quadrant (see Figure 6), which also contains the anger emotion (as per [55]).

\section{Emotions After Driving}

After having driven through an anger-inducing course, participants reported still being aroused (all mean arousal ratings were above three on a 5-Point Likert scale). There was a clear distribution of the emotions over the negative and positive valence quadrants (see Figure 7), however, this was not supported by the statistical tests. A Shapiro-Wilk test showed a significant departure from normality for all the variables in this dataset. The Kruskal Wallis test showed no statistically

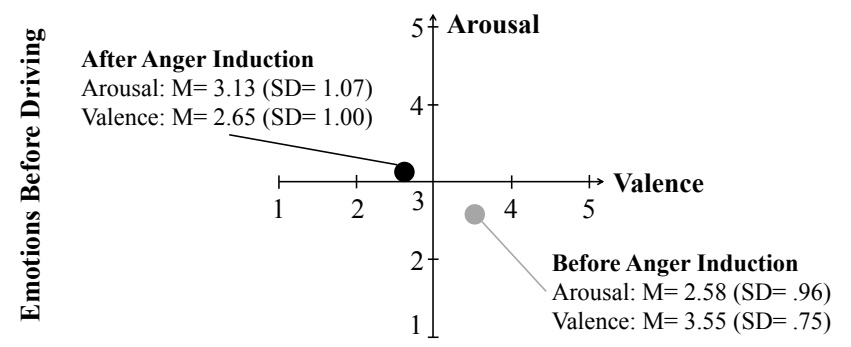

Figure 6: Mean Arousal (1= "Low", 5= "High") and Valence (1= "Negative", $5=$ "Positive") ratings of the participants' self-reported emotional state before and after the anger induction procedure, prior to the start of the driving phase. significant differences in the arousal $\left(\chi^{2}(3)=5.39, p=.145\right.$, median $=3.0,25$ th quartile $=2.0,75$ th quartile $=4.0)$ and valence $\left(\chi^{2}(3)=2.87, p=.412\right.$, median $=3.5,25$ th quartile $=3.0$, 75 th quartile $=4.0)$ ratings. We also checked for the changes of the self-reported emotions between the three points of time: before the experiment, after viewing the IAPS pictures, and after driving. The Friedman test showed a statistically significant effect of timing on the arousal $\left(\chi^{2}(1)=6.76, p<0.01\right)$ and valence $\left(\chi^{2}(1)=5.76, p<0.05\right)$ ratings. There was no significant interaction between the time and the scent for the arousal $(p=.320)$ and valence $(p=.104)$.

\section{Driving Performance and Behaviour}

The Shapiro-Wilk Normality Test showed $(p=.02)$ for the lane deviation and the steering angle, and $(p=.04)$ for the average speed. For this reason, we compared the means of the four conditions using the non-parametric Kruskal-Wallis $\mathrm{H}$ Test (as per [45]). The test showed no statistically significant differences for lane deviation $\left(\chi^{2}(3)=6.15, p=.11\right)$, steering angle $\left(\chi^{2}(3)=2.86, p=.41\right)$, and average speed $\left(\chi^{2}(3)=4.99\right.$, $p=.17)$. These results are summarised in Table 3 .

\section{Negotiating Critical Events}

While experiencing critical events (e.g. car cutting off), it was important for the participants to avoid collisions. However, only five participants out of 40 (three in rose and two in peppermint conditions) were able to complete their driving without colliding into another vehicle, a bicycle, or a pedestrian. A Shapiro-Wilk test showed a significant departure from normality for all the variables in this dataset. The Kruskal Wallis test showed statistically significant differences in the number of collisions between the scents $\left(\chi^{2}(3)=26.27, p<\right.$ .001 , median $=1.0$ ) with the highest number of collisions in the civet condition (see Figure 8). However, no significant differences were found in the binary measures of catastrophic road excursions $\left(\chi^{2}(3)=7.13, p=.068\right.$, median $\left.=.0\right)$. No excursions were recorded in the rose condition. There were two such occurrences in the water (clean air, control) and peppermint conditions. Finally, half of all 10 participants in the civet condition had experienced an excursion.

\section{Scent Comfort and Liking Ratings}

After the experiment, participants were asked to rate how much they liked interacting with the scent and how comfortable that was on a 5-Point Likert scale ( $1=$ "Not at all", 5= "Very much”). A Shapiro-Wilk test showed a significant departure

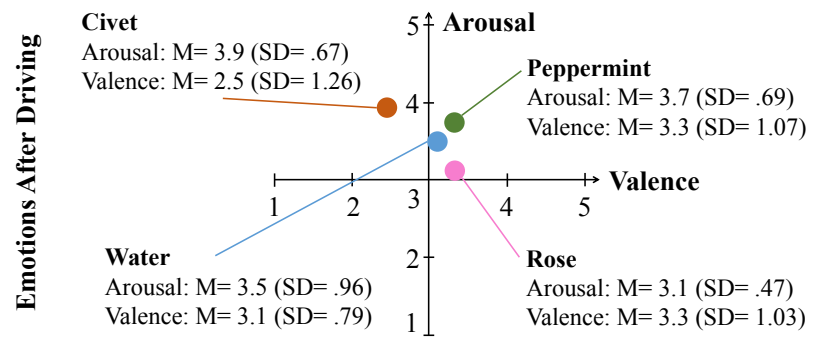

Figure 7: Mean Arousal (1= "Low", 5= "High") and Valence (1= "Negative", $5=$ "Positive") ratings of the participants' self-reported emotional state after the driving in the water (clean air), rose, peppermint, and civet conditions. 


\begin{tabular}{|l|c|c|c|c|}
\cline { 2 - 5 } \multicolumn{1}{c|}{} & \multicolumn{4}{c|}{ Condition Mean (SD) } \\
\cline { 2 - 5 } \multicolumn{1}{c|}{} & Water/Clean Air & Rose & Peppermint & Civet \\
\hline Lane Deviation $(\mathrm{m})$ & $.44(.11)$ & $.52(.26)$ & $.71(.50)$ & $.73(.33)$ \\
\hline Steering Angle $(\mathrm{rad})$ & $.09(.01)$ & $.10(.03)$ & $.09(.02)$ & $.11(.03)$ \\
\hline Average Speed $(\mathrm{mph})$ & $46.68(6.95)$ & $42.95(7.41)$ & $44.63(6.71)$ & $48.83(7.47)$ \\
\hline
\end{tabular}

Table 3: Mean scores and standard deviations of the objective driving performance and behaviour data.

from normality for all the variables in this dataset. The Kruskal Wallis test showed no statistically significant differences in the scent liking ratings $\left(\chi^{2}(3)=6.32, p=.097\right.$, median $\left.=4.0\right)$ and a significant effect of scents on the comfort ratings $\left(\chi^{2}(3)=\right.$ $7.81, p=.05$, median $=4.0$ ) with rose rated significantly higher than civet (see Figure 9).

\section{Interview Responses}

All interviews were transcribed and open coding was used to analyse the data. Below we summarise participants' impressions on the anger-induction procedure and the effects of scents on their emotions and driving.

$85 \%$ of participants had confirmed being negatively affected by the anger-inducing pictures shown at the very beginning of the experiment. These participants have described their emotional state as disturbed/distressed/upset/uncomfortable (17), angry/frustrated (16), sad (16), not happy/not positive/negative/bad (5), unpleasant/irritated/disgusted (5), disappointed/discouraged (2), confused (2), insecure (1) and stressed (1). For example, $P 20$ described their emotions in the following way: "These are not very pleasant pictures. Especially the last two were a bit disturbing because there were young kids involved in a war... They made me feel mostly angry." Those who did not report being affected by the pictures $(15 \%)$ explained this by the fact of being exposed to such content regularly. For example, $P 31$ said: "The content is really strong, but that is something I am used to seeing through news, movies, and social media."

93\% of participants had confirmed being negatively affected by the on-road events (i.e. a pedestrian suddenly crossing the road, a slow zigzagging car, a bicycle cutting off and a car cutting off) experienced in the driving simulator. They described their feelings as angry/frustrated/furious/annoyed/pissed off/irritated/impatient (25), having experienced something

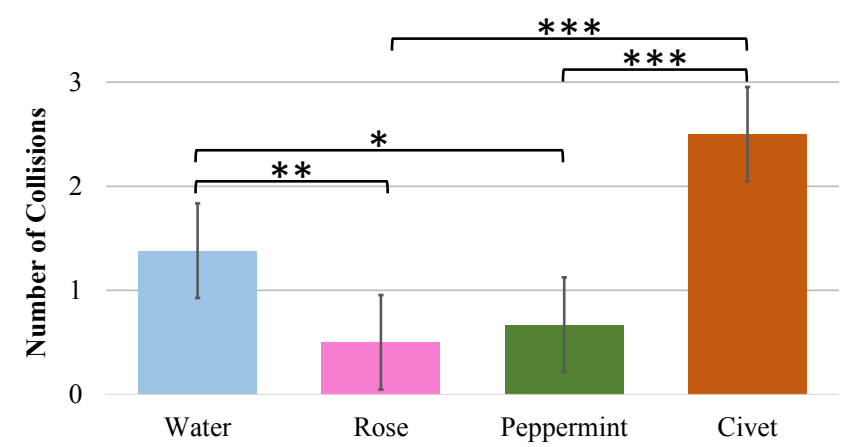

Figure 8: The mean number of collisions in the water (clean air, control), rose, peppermint, and civet conditions. Error bars, \pm s.e.m., $* p<.05$; $* * * p<.001$ unexpected/unpredictable/crazy/unsafe/random/hectic (15), stressed (7), anxious/worried (3), upset/distressed/discouraged (3), confused (2) and cautious (1). For example, P15 said: "I felt a little annoyed by the pedestrians. They were everywhere and unexpected. Drivers were like drunk, and they made me angrier than the pictures." Those, who said they did not feel affected, explained this by either knowing that they are in a safe simulator environment, or having had similar virtual experiences in the past, or generally being a very calm person. For example, P18 said: "I did not really feel anything. If they were real, where they would put my life under threat, then I am sure I would be very annoyed."

As the descriptors used by the participants lie in the "anger" (high arousal, negative valence) quadrant of the emotions circumplex [55] and the number of affected participants went up after having experienced anger-eliciting on-road events, the anger-induction procedure can be considered successful.

\section{Perceived Emotions}

Here, we summarise the interview responses on the participants' emotions during the experiment (see Figure 10).

All 10 participants who smelled rose said this scent made them feel less nervous (1), more relaxed/peaceful/settled/soothed (5), positive (1), attentive (1), positively affected (1), and less emotional (1). For example, P35 said: "It made me feel more relaxed... It was nice to have it in the car." and $P 9$ said: "It did positively affect me, it slowed me down."

6/10 participants who smelled peppermint highlighted the arousing and hedonic properties of this scent. They said that it made them positively affected (1), happy (1), more attentive/aware (3) and comfortable (1). For example, P33 said: "One thing for sure is that I was more aware of the events because of the scent." 2/10 participants claimed the scent made

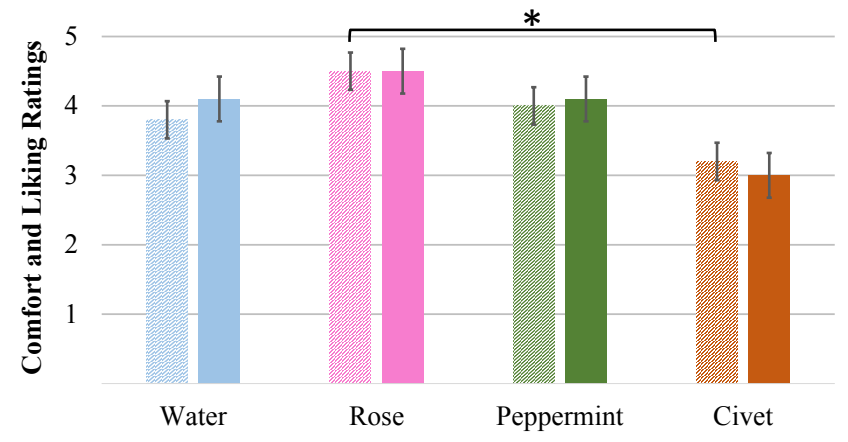

Figure 9: Mean Comfort (left bar) and Liking (right bar) ratings (1= "Not at all", $5=$ "Very much") of interacting with the scents. Error bars, \pm s.e.m., $* \boldsymbol{p}<.05$; 
Participants' emotions during the driving (interview responses):

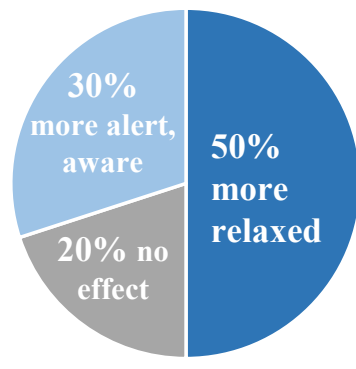

Water/Clean air

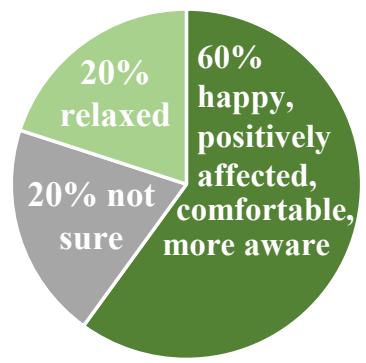

Peppermint

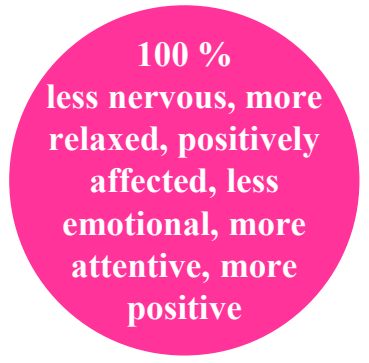

Rose

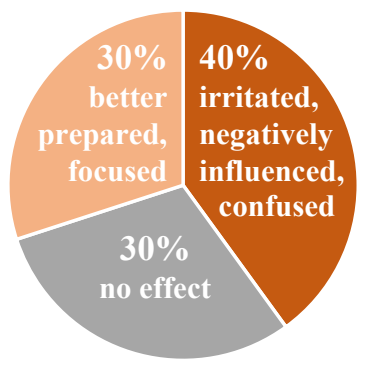

Civet
Figure 10: Percentage of participants having experienced each emotion in the process of driving.

them more relaxed because it acted as a notification and prevented them from speeding or crashing. For example, $P 11$ said: "It made me calmer. Without it I would crash four times!"

4/10 participants who smelled civet underlined the negative effect of this scent. They said that the scent made them irritated/ annoyed (2), negatively influenced (1), and confused (1). For example, P5 said: "I felt a strong negative influence. I was nervous and not relaxed.", whereas P38 said: "When I got the smell, I was really annoyed, and I wanted to finish the driving faster." 3/10 participants reported being better prepared (1), more focused (1), and able to rethink the situation (1) with the help of the scent. For example, P28 said: "There was a moment when the smell arrived just while I was passing or when I was doing a curve, and I was going too quick... That was an occasion to rethink what has happened." However, these responses do not tell us much about the participants' emotional state. Surprisingly, two of these participants reported that they liked this scent (despite the low mean liking rating).

In the control (clean air/water) condition, 5/10 participants felt more relaxed (e.g. due to fresh air). For example, $P 7$ said: "It was relaxing. It might have been just the airflow." 3/10 participants reported being more alert. For example, P17 said: "When it was coming through, I was thinking... OK, what's up ahead? It was like a warning to me."

\section{Perceived Driving Behaviour}

Here, we summarise the interview responses on the participants' perceived driving behaviour (see Figure 11).

$5 / 10$ participants who smelled rose claimed they drove in a much more considered, cautious, and focused fashion thanks
Participants' perceived driving behaviour (interview responses):
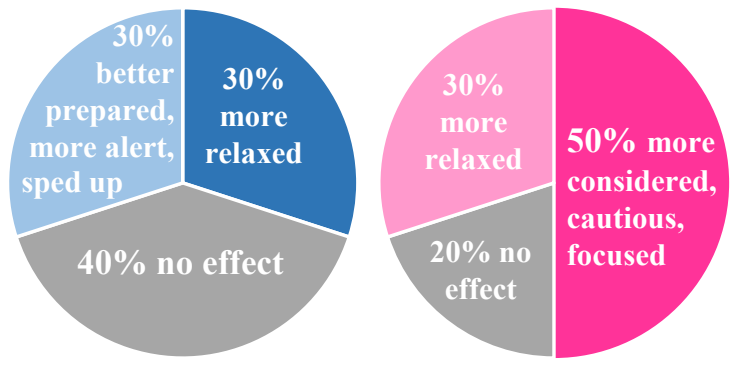

Water/Clean air

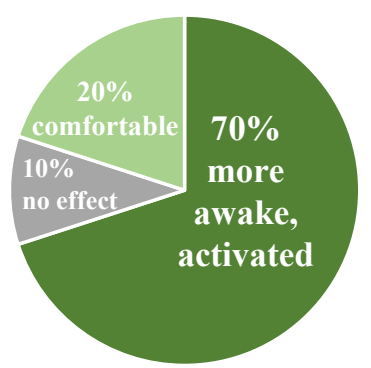

Peppermint

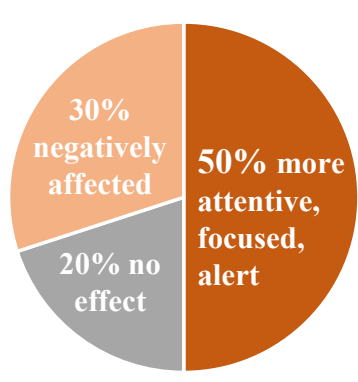

Civet
Figure 11: Percentage of participants having experienced each driving behaviour change.

to the scent, which makes sense considering its calming effect [18]. For example, P35 said: “In this particular case, I was more aware to see if there is a dangerous situation or at least a dangerous setting for a situation, like when a car would be on the side of a road, and somebody could just jump out..." 3/10 participants felt that they drove in a more relaxed way due to the scent. For example, P26 said: "It was quite a soothing and relaxing smell... It helped me relax."

$7 / 10$ participants who smelled peppermint said that the scent made them more awake and activated on the road, leading to both positive (e.g. preventing crashes) and negative (e.g. speeding) consequences. For example, P31 said: "When you are driving long ways, it could make you more aware, activate you." and P37 said: "There was one moment when I thought I drove faster when the smell came." 2/10 participants could only highlight the hedonic advantages of the scent by saying that it made their driving more comfortable. For example, P27 said: "If there is a good smell, I just feel comfortable."

What it comes to the effect of civet scent on the driving, then only 3/10 participants reported being influenced by its unpleasant properties. Participants said that they became more irritated drivers, that their driving was negatively affected, and that they were doing a lot of "illegal stuff". For example, P5 said: "The scent influenced my driving negatively, I was exceeding the speed, I was not driving smoothly; there was no positive effect." Surprisingly, 5/10 participants argued they felt that they became more attentive, focused, and alert drivers. However, since the quantitative data showed that participants had more crashes in this condition (see Figure 8), it might be that in interviews participants were relying on the arousing 
property of the civet scent, disregarding the negative valence. For sure, there is an activating effect. It is, for example, reflected in a response from P22: "When you are driving, it's very repetitive. When you get a smell, you think... Oh! It pitched me up a little bit!" However, the outcome of such a pitch might not be positive.

In the control (clean air/water) condition, 4/10 participants were not conclusive about any noticeable effect of the scent, which was expected. However, 3/10 said that it made them drive in a more alert way, be better prepared for the on-road events, and speed up. Finally, the remaining 3/10 participants found the scent relaxing. For example, P32 said: "When I got the smell, it helped me relax and do the turns a little bit better, a little bit less wobbly." This might be due to fresh air, as revealed in the responses on the emotional state.

\section{DISCUSSION}

Anger is one of the most dangerous emotions that a driver can experience, leading to aggressive driving [21] and crashes [26]. Finding a way of reducing anger might become crucial in lowering the number of traffic accidents. The results of Study 4 led to a discussion on how the scents of rose, peppermint, and civet influence the driving behaviour of participants in an induced angry state, as well as how these scents affect their emotions and well-being. We conclude with possible implications for design beyond an automotive context.

\section{Scents and Driving Behaviour}

Our study has shown that the scent of rose resulted in the lowest average speed (among all conditions) and a lane deviation that is comparable to the control condition. In the rose condition, participants also had no crashes and reported being more cautious and relaxed due to this scent. This finding is in line with the research on the relaxing effect of the scent of rose [32]. This also means that if the scent of rose is used to notify the driver about something (e.g. a "Passing by a point of interest" event [18]), then, in addition to acting as a notification modality, it could help the driver relax.

The scent of peppermint helped the participants maintain the same speed as in the control condition and the participants have reported being more activated thanks to this scent. If we think of this scent as a notification modality for angry drivers (e.g. to say "Fill gas" [18]), then it could fit also this purpose. However, as it seems to be increasing the lane deviation, it needs to be applied carefully. Potentially, it could be useful on an empty road, but not in heavy traffic. Raudenbush et al. [53] has demonstrated the scent of peppermint to increase the driver's alertness. In this study, peppermint helped participants focus on the driving task and improved their reaction time. This finding seems not to be true for angry drivers, though, as, in our study, the number of crashes was not significantly different between the peppermint and clean air conditions.

Punishment has been demonstrated to enforce correct behaviour [57]. That made us assume that a negative scent of civet might be perceived by the participants as a punishment, motivating them to be more careful on the road. However, we saw that this scent resulted in a significantly higher number of collisions. Moreover, it led to an increase in both the lane deviation and the average speed. This finding suggests that the civet scent is not a good choice for stimulating angry drivers. This finding is in line with the conclusion of Ilmberger et al. [33], who argued that strong unpleasant scents could negatively influence people's performance. Moreover, it is known that arousal through music leads to better driving performance if, and only if, the driving task is under-stimulating (arousal increased to an optimal level) [10]. This finding could explain why adding an scent with high arousal and negative valence (like civet) to an already arousing and frustrating driving environment leads to worse driving behaviour (over-stimulation). Adding a scent which counters the driving situation with low arousal and/or positive valence (like rose or peppermint) leads to better driving behaviour.

\section{Scents and Driver's Emotions}

For peppermint and rose, there was a clear shift of the selfreported emotions towards the positive valence (both in the qualitative and the quantitative data). As also expected, emotional state in the peppermint condition was rated as more aroused than in the rose condition. These findings are in line with previous work [18]. The present study confirms that arousing and calming effects of scents also work for angry drivers (i.e. reduce their negative emotional state). Both peppermint and rose could make angry drivers happier (positive valence), but they would still stay activated (high arousal).

As expected, participants in the civet condition reported the highest negative valence and the highest arousal. The interviews confirmed this with nearly half of participants describing their emotional state as highly aroused and a high negative valence. That was expected, as civet is a strong and very unpleasant scent. This is in line with previous research [38], where participants' self-reported mood in the unpleasant scent condition (e.g. dimethyl sulfide) was worse than in a pleasant scent condition (e.g. lavender). It is, however, important to consider the challenge of personal preferences here. In the interview, $20 \%$ participants in the civet condition reported liking this scent. This finding indicates, there might be drivers who choose to have this unpleasant scent in their cars. Nevertheless, the results of our study suggest that, if a driver is angry, the scent of civet might only worsen their emotions, irrespective of their personal preferences. This is important to take into account when designing experiences for angry drivers.

The arousal rating of the driver's emotions in the control condition fell between the ratings of the peppermint and rose conditions (i.e. in the higher range), and its valence stayed neutral (see Figure 7). If we compare this to the self-reported emotions before driving (see Figure 6), we can see that the valence has shifted away from the negative range. As revealed in the interviews, this might be due to an effect of fresh air, found relaxing by a half of the participants in this condition.

Our findings suggest that, when designing olfactory experiences to reduce anger in drivers, HCI practitioners should avoid civet. Peppermint and rose could help improve the driver's mood. It might be possible to achieve the same mood improvement effect by using scents of the comparable valence and arousal levels (e.g. vanilla instead of rose and lemon instead of peppermint). Such a swap might help solve the 
problem of inter-personal differences, which we also observed in our study. For example, interview responses revealed that $20 \%$ of participants in the peppermint condition claimed that this scent made them calmer (e.g. because it is associated with something "nice" and "comforting"). Therefore, we suggest developing customisable olfactory interfaces (as suggested in [63]). With such an interface, a driver could be offered a selection of positive valence scents (e.g. lavender, vanilla, rose, peppermint, lemon) and would then be asked to indicate which ones they find calming and which ones arousing/activating. This could help them avoid scents that they do not like and find scents suited for anger reduction.

\section{Scents and Well-Being of Angry Drivers}

As expected, the scents of rose and peppermint appeared to be the most suitable to increase the well-being of angry drivers, as the comfort ratings of these two scents were the highest. The scent of civet, on the contrary, had the lowest comfort rating, also correlating with the worst driving behaviour.

On this point, it is interesting to project our findings on the current trends in the automotive industry. For example, the official website of Mercedes-Benz [14] suggests that they have four different fragrances to modulate drivers' well-being, but not many details are provided. It seems though that these fragrances are sophisticated blends of odourants. In one of the recent interviews [31], Annabelle Kanzow-Coffinet, a fragrance designer of BMW [7], said that commonly used scents (e.g. lavender) are "too well known" for a car. She argued that an in-vehicle scent should be "unbiased". This statement makes sense in light of the related work on the perception of luxury experiences, which says that a luxury product (such as a high-end vehicle) should provide a unique experience associated with a unique ritual (as per [25]). With this in mind, our recommendation is to use peppermint and rose (or scents of the same arousal and valence levels) as base notes of in-car fragrances used to increase the driver's well-being. What it comes to unpleasant scents (e.g. civet), then car manufacturers would most likely not want a bad scent to be associated with their vehicles. It might, however, be that an unpleasant scent proves itself useful as a warning, motivating its choice as a suitable stimulus. This requires further investigation though.

\section{Applications Beyond Automotive Context}

Our findings can be useful also outside the context of driving a vehicle, e.g. in such areas as multisensory cinemas, gaming, VR/AR, desktop applications, and well-being wearables.

For example, if a user has had a very tense VR experience or watched an aggressive scene of an action movie in a multisensory cinema, they might need a whiff of a pleasant low arousal scent (e.g. rose) in a transition to a calmer scene. This could contribute to the research on multimedia synchronisation [47] or combining visual content with olfactory stimulation [59, 64]. On the contrary, if a player wants to have a richer gaming experience, content creators could design experiences in such a way that they regulate or modulate the user's state using scents. In such a case, even an unpleasant scent (e.g. civet) could match some of the situations in a game (as in [27, 1]). On the other note, a pleasant arousing scent (e.g. peppermint) could be used to, e.g. improve the user's well-being while maintaining their performance in a game (like in [34, 44]) or a desktop application (like in $[9,42]$ ). Finally, in case of wearable well-being devices [20,3], if the system detects that the user is angry, it could deliver a pleasant scent (e.g. rose).

\section{Limitations}

In our study, we investigated only one scent per valence and arousal quadrant. It could be that e.g. the scents of vanilla and lavender have similar effects as the scent of rose (e.g. as suggested by $[18,17])$, whereas lemon and cinnamon have similar effects as peppermint (e.g. as suggested by $[63,53])$. Also, our experiment was limited to a lab environment and a small sample size. Moreover, despite having a racing seat and a separate screen for the dashboard, our driving simulator is still quite low-fidelity. The results could have changed if a higher fidelity simulator was used. Finally, we only had 10 participants per condition which is quite a small sample size. More participants would be needed to strengthen our findings.

\section{CONCLUSION}

It is dangerous to feel angry while driving a car. Anger leads to aggressive driving behaviour [21] and crashes [26]. The 2018 data from the National Highway Traffic Safety Administration (NHTSA) [48] suggests that $94 \%$ of all road traffic accidents are caused by driver error. $33 \%$ of such accidents could be linked to behaviours associated with road rage (e.g. driving too fast, illegal manoeuvre). Therefor, a strategy for changing anger to a positive or neutral emotion might become crucial in reducing the number of accidents. Our findings suggest that pleasant scents (such as rose and peppermint) could be able to shift the emotion of the driver towards the positive valence. However, in terms of well-being, it would be necessary to customise the choice based on the driver's preferences. From its properties, the scent of peppermint is more arousing than rose, however, our interview data provides hints that some people might find it calming, as peppermint is associated with comforting experiences. In terms of driving behaviour, our findings show that an unpleasant scent (i.e. civet) might not be a good choice for stimulating angry drivers, as it results in a significantly higher number of collisions. On the contrary, pleasant scents of rose and peppermint could be able to help calm the driver down.

\section{FUTURE WORK}

In the future, we plan to extend this study by investigating other common emotions (e.g. anxiety, happiness, normal state) and scents (e.g. vanilla, lemon, cinnamon). We will also conduct a power analysis to determine the appropriate number of participants necessary to expect significant findings.

\section{ACKNOWLEDGMENTS}

The authors thank IPG Automotive for providing the CarMaker licence free of charge for conducting this research project.

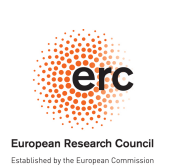

This project has received funding from the European Research Council (ERC) under the European Union's Horizon 2020 research and innovation programme under the grant agreement No 638605 and No 737576. 


\section{REFERENCES}

[1] S.H. Abid, Z. Li, R. Li, and J. Waleed. 2015. Heat Based Odors Delivery Apparatus for Interactive Game Playing. International Journal of Multimedia and Ubiquitous Engineering (2015).

[2] O. Alaoui-Ismaili, O. Robin, H. Rada, A. Dittmar, and E. Vernet-Maury. 1997. Basic emotions evoked by odorants: comparison between autonomic responses and self-evaluation. Phys. \& Beh. (1997).

[3] J. Amores, J. Hernandez, A. Dementyev, X. Wang, and P. Maes. 2018. BioEssence: A Wearable Olfactory Display that Monitors Cardio-respiratory Information to Support Mental Wellbeing. In 2018 40th Annual International Conference of the IEEE Engineering in Medicine and Biology Society (EMBC). IEEE.

[4] R.A. Baron and M.J. Kalsher. 1998. Effects of a Pleasant Ambient Fragrance on Simulated Driving Performance: The Sweet Smell of... Safety? Environment and Behavior 30, 4 (1998).

[5] K. Belkin, R. Martin, S.E. Kemp, and A.N. Gilbert. 1997. Auditory Pitch as a Perceptual Analogue to Odor Quality. Psychological Science 8, 4 (1997).

[6] Bentley. 2019. Personalising Your Bentley, and Your Life. https://www. bentleymotors. com/en/world-ofbentley/mulliner/personal-commissioning/ personalising-your-bentley.html. (2019). [Online; accessed 14-June-2019].

[7] H. Boeriu. 2015. She created the smell of the all-new BMW 7 Series. https://www.bmwblog. com/2015/07/03/ she-created-the-smell-of-the-all-new-bmw-7-series/. (2015). [Online; accessed 14-June-2019].

[8] M.M. Bradley and P.J. Lang. 1994. Measuring emotion: The self-assessment manikin and the semantic differential. Journal of Behavior Therapy and Experimental Psychiatry 25, 1 (1994).

[9] S. Brewster, D. McGookin, and C. Miller. 2006. Olfoto: Designing a Smell-based Interaction. In CHI 'O6. ACM, New York, NY, USA.

[10] P.L. Broadhurst. 1957. Emotionality and the Yerkes-Dodson law. Journal of experimental psychology 54, 5 (1957).

[11] G. Burnett, A. Hazzard, E. Crundall, and D. Crundall. 2017. Altering Speed Perception Through the Subliminal Adaptation of Music Within a Vehicle. In AutomotiveUI '17. ACM, New York, NY, USA.

[12] M. Chan and A. Singhal. 2015. Emotion matters: Implications for distracted driving. Safety Science 72 (2015).

[13] C. Chrea, D. Grandjean, S. Delplanque, I. Cayeux, B. Le Calve, L. Aymard, M.I. Velazco, D. Sander, and K.R. Scherer. 2008. Mapping the Semantic Space for the Subjective Experience of Emotional Responses to Odors. Chemical Senses (2008).
[14] Daimler. 2019. A fragrance for the new S-Class. https://www.mercedes-benz.com/en/mercedes-benz/ innovation/a-fragrance-for-the-new-s-class/. (2019). [Online; accessed 14-June-2019].

[15] D. Dmitrenko, E. Maggioni, and M. Obrist. 2017. OSpace: Towards a Systematic Exploration of Olfactory Interaction Spaces. In ISS '17. ACM, NY, USA.

[16] D. Dmitrenko, E. Maggioni, and M. Obrist. 2018. I Smell Trouble: Using Multiple Scents To Convey Driving-Relevant Information. In ICMI '18. ACM, NY, USA.

[17] D. Dmitrenko, E. Maggioni, and M. Obrist. 2019. Towards a Framework for Validating the Matching Between Notifications and Scents in Olfactory In-Car Interaction. In CHI EA '19. ACM, New York, NY, USA.

[18] D. Dmitrenko, E. Maggioni, C.T. Vi, and M. Obrist. 2017. What Did I Sniff?: Mapping Scents Onto Driving-Related Messages. In AutomotiveUI '17. ACM, New York, NY, USA.

[19] D. Dmitrenko, C.T. Vi, and M. Obrist. 2016. A Comparison of Scent-Delivery Devices and Their Meaningful Use for In-Car Olfactory Interaction. In AutomotiveUI '16. ACM, New York, NY, USA.

[20] D. Dobbelstein, S. Herrdum, and E. Rukzio. 2017. inScent: A Wearable Olfactory Display As an Amplification for Mobile Notifications. In ISWC ' 17. ACM.

[21] C.S. Dula and M.E. Ballard. 2003. Development and Evaluation of a Measure of Dangerous, Aggressive, Negative Emotional, and Risky Driving1. Journal of Applied Social Psychology 33, 2 (2003).

[22] A. Eherenfreund-Hager, O. Taubman-Ben-Ari, T. Toledo, and H. Farah. 2017. The effect of positive and negative emotions on young drivers: A simulator study. Transportation Research Part F: Traffic Psychology and Behaviour 49 (2017).

[23] S.M. Fakhrhosseini, S. Landry, Y.Y. Tan, S. Bhattarai, and M. Jeon. 2014. If You'Re Angry, Turn the Music on: Music Can Mitigate Anger Effects on Driving Performance. In AutomotiveUI '14. ACM, NY, USA.

[24] H. Funato, M. Yoshikawa, M. Kawasumi, S. Yamamoto, M. Yamada, and Y. Yanagida. 2009. Stimulation effects provided to drivers by fragrance presentation considering olfactory adaptation. In Intelligent Vehicles Symposium 2009. IEEE.

[25] V. Grigorian and F.E. Petersen. 2014. Designing Luxury Experience. ESMT 14, 04 (2014).

[26] A.C. Hayley, B. de Ridder, C. Stough, T.C. Ford, and L.A. Downey. 2017. Emotional intelligence and risky driving behaviour in adults. Transportation Research Part F: Traffic Psychology and Behaviour (2017).

[27] N.S. Herrera and R.P. McMahan. 2014. Development of a simple and low-cost olfactory display for immersive media experiences. In ImmersiveMe'14. ACM. 
[28] R.S. Herz and T. Engen. 1996. Odor memory: Review and analysis. Psychonomic Bulletin \& Review 3, 3 (1996).

[29] R. S. Herz. 2002. Influences of Odors on Mood and Affective Cognition. In Olfaction, Taste, and Cognition. Cambridge University Press.

[30] S. Hiroike, S. Doi, T. Wada, E. Kobayashi, M. Karaki, N. Mori, T. Kusaka, and S. Ito. 2009. Study of olfactory effect on individual driver under driving. In ICME '09.

[31] F. Hoberg. 2019. Dufttricks der Autoindustrie: Morgens riecht der Wagen nach Meer, abends nach Wüste. https: //www. spiegel.de/auto/fahrkultur/bmw-mercedes-und-cowie-hersteller-innenraeume-beduften-a-1272068.html. (2019). [Online; accessed 13-June-2019].

[32] T. Hongratanaworakit. 2008. Relaxing Effect of Rose Oil on Humans. Natural Product Communications (2008).

[33] J. Ilmberger, E. Heuberger, C. Mahrhofer, H. Dessovic, D. Kowarik, and G. Buchbauer. 2001. The influence of essential oils on human attention. I: Alertness. Chemical Senses 26, 3 (2001).

[34] Y. Ishibashi, S. Hoshino, Q. Zeng, N. Fukushima, and S. Sugawara. 2012. QoE assessment of fairness between players in networked game with olfaction. In Proceedings of the 11th Annual Workshop on Network and Systems Support for Games. IEEE Press.

[35] M. Jeon, B.N. Walker, and J.B. Yim. 2014. Effects of specific emotions on subjective judgment, driving performance, and perceived workload. Transportation Research Part F: Traffic Psych. and Beh. (2014).

[36] M. Jeon, J.B. Yim, and B.N. Walker. 2011. An Angry Driver is Not the Same As a Fearful Driver: Effects of Specific Negative Emotions on Risk Perception, Driving Performance, and Workload. In AutomotiveUI'11. ACM, New York, NY, USA.

[37] R.M. Khan, C.H. Luk, A. Flinker, A. Aggarwal, H. Lapid, R. Haddad, and N. Sobel. 2007. Predicting Odor Pleasantness from Odorant Structure: Pleasantness as a Reflection of the Physical World. J. Neuroscience 27, 37 (2007).

[38] S.C. Knasko. 1992. Ambient odor's effect on creativity, mood, and perceived health. Chemical senses 17, 1 (1992), 27-35.

[39] P.J. Lang. 2005. International affective picture system (IAPS) : affective ratings of pictures and instruction manual. Technical Report (2005).

[40] John D. Lee, Joshua D. Hoffman, and Elizabeth Hayes. 2004. Collision Warning Design to Mitigate Driver Distraction. In $\mathrm{CHI}$ '04. ACM, USA.

[41] E. Maggioni, E. Agostinelli, and M. Obrist. 2017. Measuring the added value of haptic feedback. In QoMEX' 17.
[42] E. Maggioni, R. Cobden, D. Dmitrenko, and M. Obrist. 2018. Smell-O-Message: integration of olfactory notifications into a messaging application to improve users' performance. In ICMI '18. ACM, New York, NY, USA.

[43] G.N. Martin and J.A. Cooper. 2007. Adding zest to difficult journeys: Odour effects on simulated driving performance. In The British Psychological Society Annual Conference.

[44] A. Mochizuki, T. Amada, S. Sawa, T. Takeda, S. Motoyashiki, K. Kohyama, M. Imura, and K. Chihara. 2004. Fragra: a visual-olfactory VR game. In $A C M$ SIGGRAPH 2004 Sketches. ACM.

[45] B. Mok, M. Johns, D. Miller, and W. Ju. 2017a. Tunneled In: Drivers with Active Secondary Tasks Need More Time to Transition from Automation. In CHI '17. ACM, New York, NY, USA.

[46] B. Mok, M. Johns, S. Yang, and W. Ju. 2017b. Reinventing the Wheel: Transforming Steering Wheel Systems for Autonomous Vehicles. In UIST'17. ACM, New York, NY, USA.

[47] N. Murray, G.M. Muntean, Y. Qiao, and B. Lee. 2018. Olfaction-Enhanced Multimedia Synchronization. Springer International Publishing.

[48] NHTSA. 2018. Critical Reasons for Crashes Investigated in the National Motor Vehicle Crash Causation Survey. https://crashstats.nhtsa.dot.gov/ Api/Public/ViewPublication/812506. (2018). [Online; accessed 07-Jan-2020].

[49] D. Noguchi, S. Sugimoto, Y. Bannai, and K. Okada. 2011. Time Characteristics of Olfaction in a Single Breath. In CHI '11. ACM, NY, USA.

[50] K. Ohtsu, J. Sato, Y. Bannai, and K. Okada. 2009. Scent Presentation Technique of Pulse Ejection Synchronized with Breathing. In Ninth Annual International Symposium on Applications and the Internet, 2009. SAINT'09.

[51] C. Oshima, A. Wada, H. Ando, N. Matsuo, S. Abe, and Y. Yanigada. 2007. Improved delivery of olfactory stimukus to keep drivers awake. In Workshop on DSP for in-Vehicle and Mobile Systems.

[52] I. Politis, S. Brewster, and F. Pollick. 2015. To Beep or Not to Beep?: Comparing Abstract Versus Language-Based Multimodal Driver Displays. In CHI '15. ACM, New York, NY, USA.

[53] B. Raudenbush, R. Grayhem, T. Sears, and I. Wilson. 2009. Effects of Peppermint and Cinnamon Odor Administration on Simulated Driving Alertness, Mood and Workload. N.A.J. of Psychology (2009).

[54] E. Roidl, B. Frehse, and R. Höger. 2014. Emotional states of drivers and the impact on speed, acceleration and traffic violations-A simulator study. Accident Analysis and Prevention (2014). 
[55] J.A. Russell. 1980. A circumplex model of affect. Journal of personality and social psychology 39, 6 (1980).

[56] S.R.H. Sani, Z. Tabibi, J.S. Fadardi, and D. Stavrinos. 2017. Aggression, emotional self-regulation, attentional bias, and cognitive inhibition predict risky driving behavior. Accident Analysis and Prevention 109 (2017).

[57] J.B. Sidowski, L.B. Wyckoff, and L. Tabory. 1956. The influence of reinforcement and punishment in a minimal social situation. The Journal of Abnormal and Social Psychology 52, 1 (1956).

[58] D. Sirkin, N. Martelaro, M. Johns, and W. Ju. 2017. Toward Measurement of Situation Awareness in Autonomous Vehicles. In CHI'17. ACM, New York, NY, USA.

[59] Risa Suzuki, Shutaro Homma, Eri Matsuura, and Ken-ichi Okada. 2014. System for Presenting and Creating Smell Effects to Video. In ICMI'14. ACM, New York, NY, USA.

[60] C. Velasco, D. Balboa, F. Marmolejo-Ramos, and C. Spence. 2014. Crossmodal effect of music and odor pleasantness on olfactory quality perception. Frontiers in Psychology 5, 1352 (2014).

[61] G. Wilson and S.A. Brewster. 2017. Multi-moji: Combining Thermal, Vibrotactile \& Visual Stimuli to
Expand the Affective Range of Feedback. In CHI '17. ACM, New York, NY, USA.

[62] G. Wilson, D. Dobrev, and S.A. Brewster. 2016. Hot Under the Collar: Mapping Thermal Feedback to Dimensional Models of Emotion. In $\mathrm{CHI}$ '16. ACM, New York, NY, USA.

[63] P. Wintersberger, D. Dmitrenko, C. Schartmüller, A.K. Frison, E. Maggioni, M. Obrist, and A. Riener. 2019. S(C)ENTINEL: monitoring automated vehicles with olfactory reliability displays. In IUI '19. ACM, New York, NY, USA.

[64] B. Wu, Q.H. Meng, H.R. Hou, M. Zeng, and P.F. Qi. 2018. An Olfactory Display System Integrated with Video Content Recognition. In 2018 13th World Congress on Intelligent Control and Automation (WCICA). IEEE, 1641-1646.

[65] Y. Yanagida, S. Kawato, H. Noma, A. Tomono, and N. Tesutani. 2004. Projection based olfactory display with nose tracking. In Virtual Reality, Proceedings. IEEE.

[66] M. Yoshida, C. Kato, Y. Kakamu, M. Kawasumi, H. Yamasaki, S. Yamamoto, T. Nakano, and M. Yamada. 2011. Study on Stimulation Effects for Driver Based on Fragrance Presentation. In MVA2011 IAPR. 